\title{
Arrangement of muscle fibers in the myometrium of the human uterus: a mesoscopic study
}

\begin{abstract}
The human uterus is a hollow, thick-walled and contractile organ with the purpose of receiving the product of fertilization along with its nesting, growth and subsequent birth. Part of its function is made possible due to its muscular layer, traditionally described as consisting of three layers or strata: an outer layer of predominantly horizontal and then longitudinal fibers; a middle layer comprised of circular fibers arranged in multiple directions containing a layer of blood vessels; and an inner layer of similar structure to the outer layer. This study aims to describe the mesoscopic way in which the muscle fibers of human uterine myometrium are arranged. 10 samples of bisected human uteri were investigated. The results following dissection first showed the perimetrium under a lower layer of longitudinal fiber followed by horizontal fibers that, as they approach the margin, become skewed in the direction of the oviducts. Also, a small amount of fibers that change from horizontal to longitudinal near the median plane were found to pass through the middle region of the body of the uterus. Beyond the deep plane, we found fibers arranged in multiple directions, resembling eddies that are directed at an angle from the outside inwards and mark the location of small blood vessels. In the deepest part, we found a thin layer containing few horizontal fibers.
\end{abstract}

Keywords: uterus, myometrium, muscle fibers
Volume 4 Issue 2 - 2017

\author{
Nelson M Escalante M,' Jorge Henríquez \\ Pino ${ }^{2}$ \\ 'Facultad de Medicina, Universidad de Talca, Chile \\ ${ }^{2}$ Facultad de Medicina, Universidad de La Frontera, Chile
}

Correspondence: Jorge Henríquez Pino, Facultad de Medicina, Universidad de La Frontera,Temuco, Chile,

Email jorge.henriquez@ufrontera.cl

Received: July 10, 2017 | Published: September 07, 2017

\section{Introduction}

There is a significant amount of information in different anatomy texts that similarly describe the myometrium. The human uterus is a hollow organ composed of thick, contractile walls intended for the implantation and growth of the product of sexual fertilization. Its function is accomplished with its muscular layer, which is largely formed by three strata of fibers, each of which is arranged in a different direction and differ and are described in a specific way. Nevertheless, there are small differences among the descriptions. Due to these variations, we decided to conduct our own research.

The first studies on uterine structure were performed by Bichat ${ }^{1} \&$ Cruveilhier ${ }^{2}$ but the most detailed and precise study was later made by Goerttler. ${ }^{3}$ After these studies, we found various descriptions in different anatomy texts which were similar to the arrangement described by Goerttler, who effectively explained the structure of the myometrium from a functional point of view. Macroscopically, the uterus is composed of a body, an isthmus and a cervix. Its anatomical constitution is formed by three overlapping tunics that are from outside to inside: a serosatunic, a muscular tunic and a tunica mucosa. The muscular tunic is mostly composed of smooth muscle fibers, which together constitute the so-called myometrium.

The smooth fibers that compose the myometrium are separated by connective-elastic trabeculae and constitute the largest part of the uterus. Its distribution in the body of the uterus ${ }^{4}$ is described as being composed of two layers, one external and one internal, with an intermediate layer between them. The outer and inner layers appear to correspond to the primary layers of the paramesonephric ducts. The fibers of the first are mostly longitudinal while those of the second are mostly circular. However, this usual arrangement is hidden by numerous bundles arranged in an intermediate direction. The middle layer, which forms at least two-thirds of total uterine thickness, appears to be a secondary formation, both phylogenetically and ontogenically. Here, the fibers are arranged around the numerous blood vessels, and are arranged in a system of plexiform sheaths that defies systematization. At the cervix, the muscular fibers are much less numerous.

Upon reviewing the literature, we realized that many authors describe the structure of the myometrium in a similar way, ${ }^{5-10}$ among others, explain the 3 layers of fibers in the structure of the myometrium, emphasizing the middle layer's larger thickness, the types of fibers arranged in circular or oblique directions, the presence of blood vessels and the complexity in describing the distribution pattern of said fibers. An important concept pointed out by Goerttler ${ }^{3}$ is the fact that it is difficult to give a complete and isolated examination of the fibers as there are multiple intersections. It is important to mention that this muscular system contributes to the formation of the ligamentous system that attaches to the uterus, ${ }^{2,6,11}$ and also highlights the importance of the superficial fibers of the myometrium that adhere strongly to the perimeter and form the wide ligament fixation system. The myometrium's composition at the cervix is described as being composed of fibers arranged horizontally, contributing to the formation of the system of sphincters at the cervical level.

The objective of this study is to offer a new description of the distribution of the myometrial fibers, indicating their directional changes in the superficial, median, and deep planes, which have not yet been described by other researchers.

\section{Materials and methods}

Ten sample of human uteri, divided in half, where provided by the Department of Pathology at the Hospital Regional of Temuco, Chile. All uteri were obtained through the informed consent of the patients. The samples belonged to Chilean women between the ages of 42 and $56 y e a r s$ who had undergone a hysterectomy. The uteri were soaked in $10 \%$ formalin for 5 days and then moved to a $5 \%$ formalin solution 
in which they were kept until mesoscopic dissection began. Next, the samples were macroscopically dissected and loose portions of the peritoneum, blood vessels, uterine tubes and ovaries were removed in order to have a better view of their external structure as well as to more easily take their respective measurements with a manual caliper (Vernier) with an accuracy of $0.05 \mathrm{~mm}$.

After their macroscopic dissection and being soaked in 5\% formalin, the samples were placed in $0.5 \%$ chromic acid for 24 hours in order to macerate the muscle fibers. After 24hours, they were removed from $0.5 \%$ chromic acid and washed with double distilled water. The dehydration process was then initiated and the samples were treated with $50^{\circ} \mathrm{C}, 70^{\circ} \mathrm{C}$ and $80^{\circ} \mathrm{C}$ alcohol for 60 minutes each and then with $95^{\circ} \mathrm{C}$ alcohols and $100^{\circ} \mathrm{C}$ for 45 minutes each. After the dehydration process, the samples were placed in pure turpentine for 24hours, and allowed to dry for 24hours. Once they hardened, the researchers proceeded to dissect with the aid of a stereoscopic magnifying glass and the naked eye.

For this dissection, caliper, scalpel handles 3 and 4 and scissors were used. Over the course of the dissection, $10 \mathrm{mp}$ format photographs were taken, with increases up to $14 \mathrm{mp}$ in high quality, showing the layers and direction of the fibers. The dissection was performed on one side of the uterus, using the median plane as a starting point. From there, the direction of the fibers was followed continuing towards the regions of the isthmus, cervix and uterine fundus.

\section{Results}

In the first sample, we identified the surface perimeter as smooth, dark and bright in color with a tartar texture. The muscular layer showed a lighter color, and the mucous layer was with a whitish color. After the dissection of all human uteri samples, we observed that the myometrium is the thickest layer of the uterus and shows the greatest thickness along the body of the uterus and at the fundus.

The perimeter was dissected, and as it was removed, a series of longitudinal muscular fibers attached to it were also removed. After dissecting most of the perimeter, we found a remarkable amount of horizontal fibers mixed with some sparse longitudinal fibers in $100 \%$ of the samples. These horizontal fibers follow a path beginning in the median plane and moving towards the uterine margin. As they approach the uterine margin, they change direction and become oblique towards the region of the uterine tubes (Figure 1). It should be noted that a small amount of fibers near the median plane change from a longitudinal to horizontal position in the middle region of the organ.
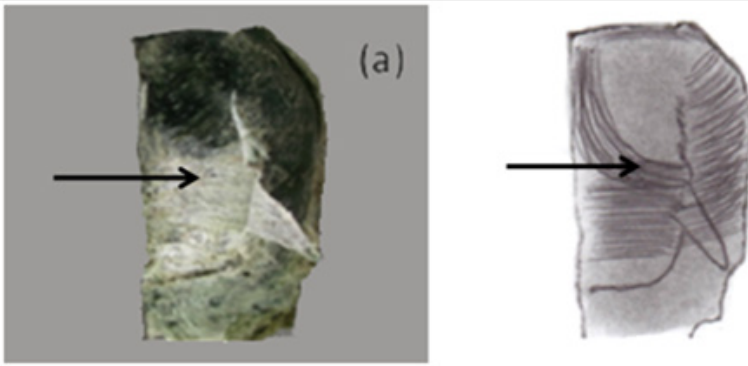

(b)

Figure I Anterior view of the uterus sectioned in the middle. (A) Horizonta fibers under the perimeter that is partially separated. The arrow indicates fibers that close to the median plane change from longitudinal to horizontal (B) Horizontal fibers are drawn, which, when approaching the margin of the uterus, become oblique, towards the uterine tubes. In the part near the uterine fundus, some fibers that have a longitudinal path, change their path to horizontal.
In the uterine fundus, fibers were longitudinal, and this continued to the posterior face of the uterus. In the same way, horizontal fibers and another group of longitudinal fibers were observed at the top, which is close to the middle plane, and shifted to a horizontal position in the upper part of the bottom, towards the region of insertion of the uterine tube. On the other hand, at the uterine cervix, only horizontal fibers were observed in $100 \%$ of the samples (Figure 2). These fibers maintained this direction as the dissection continued showing minimal changes in composition
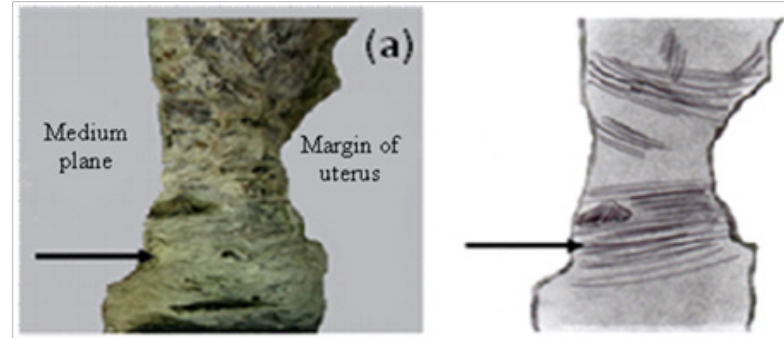

Figure 2 Anterior view of the uterus sectioned at the middle of the neck. (A) The presence of fibers in the horizontal direction is evident. This pattern was maintained throughout the dissection as we deepened. (B) The diagram shows fibers in the horizontal direction, which go from side to side.

As the dissection went deeper, fibers at the level of the body of the uterus were found to be arranged in multiple directions, resembling eddies, which were directed obliquely from the outside inwards. Among these fibers we identified some small vessels that were affected by the directional variability. Here, the dissection was rather complicated. However, at this level, the fibers had a concentric appearance around the uterine tube (Figure 3). At the cervix, we continued to find horizontal fibers.
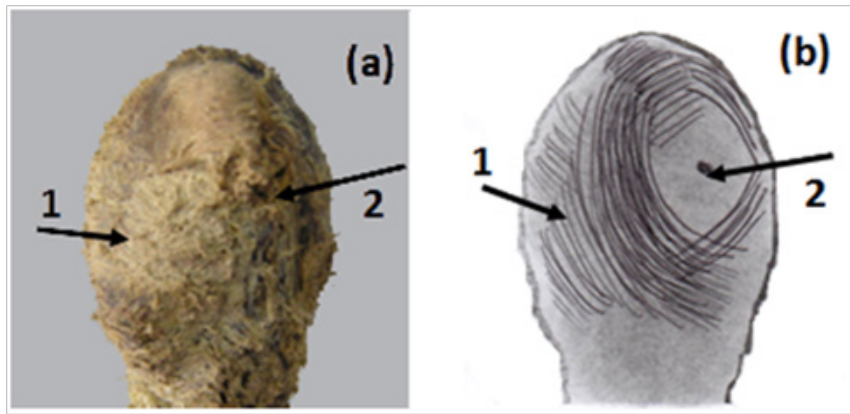

Figure 3 Anterolateral view of the uterus. (A) Circular fiber layer. Note at the start of this layer the fibers change their linear pattern and become a circular pattern (I) in relation to the uterine tube. Note the region of insertion of the uterine tube (2). (B) The illustration shows the existence of fibers circularly around the uterine tube that intersect with each other in several directions.

We continued dissecting this layer of fibers in multiple directions, which turned out to be the thickest layer. We continued to dissect the uterine wall, which become thinner and the fibers arranged in various directions became more persistent in all ten samples. The dissection of the deeper parts of the uterus became more difficult as the fibers were even more resistant to separation. However, when we began to reach the end, practically reaching the level of the endometrium, we were able to find a new pattern of fibers, which were horizontal. This layer was very thin and we could only examine this layer in the regions near the median plane of the uterus. This was because the fibers in the lateral region of the anterior layer, those arranged concentrically, were difficult to separate, a major reason why the behavior of this layer could not be fully described. 


\section{Discussion}

Our dissection of the samples demonstrated that, within the human uterus, the myometrium represents the thickest part of the uterus and is composed of muscular fibers that form continuous systems arranged in spirals of variable direction and length that are able to increase their capacity and volume without placing pressure on the fetus. ${ }^{12}$

In the first step of dissection, the dissected perimeter, when removed, brought with it fibers from the first layer of the myometrium. As Orts Llorca ${ }^{13}$ noted, this shows the strong attachment of the perimeter to the myometrium. Similarly, Spalteholz indicated that this adhesion is much more intense in the bottom and in the largest part of the body. Likewise, it is evident that some of these longitudinal fibers, in the region of the bottom and near the zone of insertion of the uterine tube, became horizontal, as described by Sala L, ${ }^{6}$ Testut L \& Latarjet $A^{7}$ Quiroz $\mathrm{G}^{10}$ Prives $\mathrm{M}$ et al. ${ }^{14}$ This refers to the first layer of the longitudinal fibers and how they behave in the horn and the uterus while also indicating that the fibers appeared to continue from the front to the back forming a kind of horseshoe shape (Hélie's ansiform fasciculus). Cruveilhier ${ }^{2}$ on the other hand, described this outer layer as consisting of vertical fibers that are the product of the straightening of the oblique fibers near the uterine tubes. In addition, Tourris et al. ${ }^{15}$ as well as Chiarugi ${ }^{5}$ \& Tandler $^{11}$ added that part of this layer of longitudinal fibers, in addition to their convergence in the region of the uterine tubes, become part of the round and utero-ovary ligaments. Rouviere \& Delmas ${ }^{16}$ indicated that in the first muscular layer there were only small amounts of longitudinal fibers that covered only the surface and fundus of the uterus. This does not describe the behavior that other authors referenced regarding the behavior near the uterine tubes and its oblique change of direction, a characteristic we observed during dissection.

The second type of fiber in the most superficial layer were the transverse fibers that seemed to go from side to side with some of them appearing to be discontinuous (likely becoming part of the perimeter and ligament system). This observation is described by Testut L \& Latarjet $A,{ }^{7}$ Quiroz $\mathrm{G}^{10}$ who indicated that the transverse fibers are directed from one side to the other of the organ, following a horizontal or slightly oblique direction. Rouviere \& Delmas, ${ }^{16}$ however, noted that the fibers found more deeply in the tissue than the longitudinal fibers are circular. Finally, the dissected fibers near the cervix were predominantly horizontal and sparse, as cited by Sala L, ${ }^{6}$ Testut L \& Latarjet $\mathrm{A}^{7}$ This indicates that, at the level of the cervix, the arrangement of these transverse fibers is very simple. Tourris et al. ${ }^{15}$ described the entire cervix region as being composed of essentially circular fibers.

Once the horizontal fibers were dissected, we then found a layer of predominantly circular fibers, except for the region of the cervix where they remained horizontal. This layer of circular fibers was directed concentrically relative to the uterine tube and the fibers appeared to originate around it. As they approached the median plane, more and more of these circular fibers intersected with other fibers arranged in multiple directions. That pattern is more difficult to schematize and was predominant as the dissection went deeper. This pattern of circular fibers is described by Cruveilhier $\mathrm{J},{ }^{2}$ Goerttler $\mathrm{K},{ }^{3}$ Chiarugi G, ${ }^{5}$ Sala L, ${ }^{6}$ Testut L \& Latarjet A, ${ }^{7}$ Spalteholz W, ${ }^{9}$ Tandler J, ${ }^{11}$ Costacurta L, ${ }^{12}$ Tourris $\mathrm{H},{ }^{15}$ Rouviere H. ${ }^{16}$ Our findings coincided in some ways with the aforementioned studies, such as the circular fiber layer being the most voluminous and being widely vascularized $^{2,4,5,11,15}$ emphasize that the distribution of this layer defied all attempts to systematize it and is almost indescribable. Poirier et al. ${ }^{4}$ added that the fibers at the neck are less numerous. However, ${ }^{5,6}$ noted that these fibers at the cervix level (given their arrangement) form the internal sphincter. It is important to note that in this layer, also called the "vascular stratum", the muscular fibers surround the blood vessels forming a sort of muscular ring, as mentioned by Testut \& Latarjet. These rings likely fulfill an important function after delivery and, at the time of delivery, they likely help prevent bleeding by compressing the blood vessels. As stated by Tourris et al., ${ }^{15}$ given that muscular fibers retract in the postpartum uterus by decreasing their length and increasing their diameter, it is clear that this plexiform layer serves a fundamental role in preventing postpartum bleeding.

\section{Conclusion}

The results of this study led us to conclude that the innermost stratum was difficult to dissect in its entirety as many of the fibers in the circular layer could not be detached, particularly those located along the uterine margin. However, a series of horizontal fibers could be observed beneath the circular fibers near the median plane. These findings do not agree with those studies which cite the presence of longitudinal fibers in the innermost layer, a layer that we were unable to identify.

We acknowledge the limitations of our mesoscopic study. Future studies could improve upon our methods by using scanning electron microscopy techniques in order to provide new insights into the arrangement of muscle fibers in the uterine myometrium.

\section{Acknowledgements}

None.

\section{Conflict of interest}

Author declares that there is no conflict of interest.

\section{References}

1. Bichat X. Traité Danatomie descriptive. París Bros Son Libraire; 1803. $638 \mathrm{p}$.

2. Cruveilhier J. Tratado de Anatomía Descriptiva. Madrid, Spain; 1851.

3. Goerttler K. Structure of the human uterus wall. Arch Gynakol. 1968;205(4):334-342.

4. Poirier P, Cuneo B, Charpy A. Tratado elemental de Anatomía Humana. Imprenta y Libreria Moya, Madrid, Spain; 1908. 467p.

5. Chiarugi G. Istituzioni di Anatomia Delluomo. 2nd ed. Societá Editricie Libraria, Milano, Italy; 1924.

6. Sala L. Apparecchio Urogenitale. In: Bertelli D, editor. Trattato di Anatomia Umana. 2nd ed. Milano: Francesco Vallardi; 1932.

7. Testut L, Latarjet A. Tratado de Anatomía Humana. 8th ed. Barcelona, Spain: Salvat Editores; 1932.

8. Testut L, Jacob O. Tratado de Anatomía Topográfica. 8th ed. Barcelona, Spain: Salvat Editores; 1964.

9. Spalteholz W. Atlas de Anatomía Humana. Barcelona, Spain; 1973.

10. Quiroz G. Tratado de Anatomía Humana. 32nd ed. Mexico: Editorial Porrua; 1993.

11. Tandler J. Tratado de Anatomía Sistémica. Barcelona, Spain: Salvat Editores; 1928.

12. Costacurta L. Anatomía medico cirúrgica da pelve humana. Sao Paulo, Brazil: Atheneu Editora; 1982.

13. Orts Llorca F. Anatomía Humana. 3rd ed. Barcelona: Editorial Científico, Médica; 1967. 
14. Prives M, Lisenkov N, Bushkovich V. Anatomía Humana. 5th ed. Moscú: Editorial Mir; 1984.

15. Tourris H, Kamina P, Rideau Y. Contribution to the study of the structure of the Miometre. Bull Assoc Anat. 1972;155:1248-1256.
16. Rouviere H, Delmas A. Anatomía Humana. 11th ed. Masson, Barcelona, Spain; 2005. 